\section{H. Takeda}

Department of Medical information Science, Osaka University Medical Hospital, Osaka, Japan

As the medical infrastructure for computing and communication develops and as computers play a more important role than ever before, data acquisition, storage, retrieval and processing are about to improve health and clinical management. The integration of health data and decision support has become much easier and much research has been carried out in this area. In this section of selected articles, major scientific projects in building and evaluating useful and acceptable health and clinical management systems are introduced and discussed.

In the first paper, "PC-based system for an objective quantification of manual movement disability for clinieal and scientific purposes" [1], Machetanz and co-authors introduce new aspects of the use of a computerhased data entry system as a quantitative measurement tool in a clinical consultation setting. Using standard hardware with a Microsoft-compatible mouse, the strategy of the revarch project was to evaluate manual movement disability. Data such as reaction/movement time, pursuit tracking, sequential movements and tapping were acquired in a homogenous Ifn using a mouse. Parameters to pucidate manual movement disability were obtained in the form of general measures of error, cross-correlation, vershoot quotient and reaction/move-

\title{
Synopsis
}

\section{Health and Clinical Management}

ment time ratio. The PC-based system seems to be clinically useful and costeffective. Clinical data collection and its assessment will be helpful in promoting this kind of research for clinical consultation.

The second paper deals with the classification of diseases by means of neural networks. In "Application of neural networks to the classification of giant cell arthritis"'[2], Astion and co-authors applied a backpropagation network to classify certain types of arthritis. Their method was applied to the analysis of a vasculitis database containing 807 cases in which 214 cases were diagnosed as giant cell arthritis (GCA) and 593 cases as other forms of vasculitis. The system correctly classified $202(94.4 \%$ ) out of the 214 GCA cases, and 545 (91.9\%) of the 593 other forms of vasculitis. Neural nets and classification trees were compared by ROC analysis. The ROC curves indicated which of the two methods gave a better classification. Although many researchers dealing with neural networks have been partially successful in developing diagnostic classification rules, clinicians are not completely satisfied with the level that was accomplished. We should keep in mind that classifying diseases and making a primary diagnosis is sometimes only a working hypothesis for further examinations to diagnose a disease. In order to facili- tate neural network related research, another clinical management model, which links the diagnosis to the therapeutic process may be required.

In Linnarsson's "Drug interactions in primary health care" [3], an analysis is reported of drug prescriptions, and the use of databases for supporting decisions in routine clinical practice is described. The objective of this study was to investigate potential drug interactions in primary health care by means of computerized medical record systems. After each drug prescription was entered into a computer-based medical record, the potential interactions of the drugs were analyzed over a fouryear period (including 55,000 prescriptions). Results indicated that potential drug interactions were found in $1.9 \%$ of all prescriptions, that $22 \%$ of elderly patients were at risk (those receiving more than two drugs), and that in many cases prescribing physicians did not take appropriate actions, despite these potential interactions. What this study shows is anew promising method for analyzing retrospective clinical data from computer-based patient record systems with the help of a query language, in order to support decision making in routine clinical practice, such as drug prescribing. These results were used to provide a basis for awareness on the side of primary health care concerning potential drug interactions. 
"Clinical databases and critical care research" by Kahn et al. [4] reported on electronic databases for clinical research in critical care. The issues discussed in this paper help to increase the awareness of clinical investigators concerning the strengths and weaknesses of the electronic storage of data in generic databases, clinical databases, and electronic medical record systems. Although the advantage of a database management system is well understood, the author stressed the problems faced by most hospitals. Because the original intentions of the databases were department oriented, most clinical database systems were limited in satisfying investigative needs, such as sharing data among various specialized systems. The key to successfully integrating a widespread patient careoriented database system is described requiring the use of standards, validated data definitions, and an adequate hardware and software environment. If the authors had had more illustrative examples concerning the handling of electronic data storage in promoting clinical research, the article would have revealed a better understanding of this topic.

The next two papers focus specifically on the use of computer-based literature searches and their impact on patient care. The objective of Gorman and co-authors' study in "Can primary care physicians' questions be answered using the medical journal literature?"[5], was to determine the proportion of medical questions generated in primary care and answerable through information obtained by literature search. A second goal was to compare time and cost of such information gathering with the current methods of information retrieval employed by general practitioners. The study was done in the form of office interviews, on-line searches by librarians, and feedback by clinicians. Results showed that the search process took $43 \mathrm{~min}$. and cost $\$ 27$ on average, and that clinicians found $46 \%$ "clear answers" to their questions, and rated the impact of such literature searches as $40 \%$ related to their patients and $51 \%$ to their practice. The study gave an evaluation of on-line medical journal literature search. If persons carrying out literature searches have the necessary expertise, and the required hardware and software, and if the time and cost of searching can be reduced, primary-care physicians are interested in accessing information via on-line medical literature databases.

In "Effect of on-line literature searching on length-of-stay and patient care costs" [6], Klein and coworkers studied the effect of literature searches using MEDLINE upon the length-of-stay and the cost of hospitalization. An outcome-based and prospective study of 192 test cases and 10,409 control cases, was conducted. The results indicated that inpatients for whom searches were conducted beforehand had significantly lower costs and length-of-stay than those for whom searches were conducted later on. Thus, the study demonstrated that an extensive "intervention" of a librarian-meditated MEDLINE search would help hospitals in optimizing the use of its resources.

The last two papers clearly demonstrate that librarians offer an original contribution to medical informatics through database searching for health and medical management.

The needs for health and medical care are increasing in quantity, but diversifying in quality. With this bad ground, health and clinical manage ment are becoming increasingly de pendent on the effective use of dati communication and information tect. nology. Researchers in the medict informatics community need to aware of this rapidly changing situa tion and they need to construct model that effectively fulfil the needs of cli nicians while properly using the nem technology. Not only do they have to develop such systems, they also have to assess their impact. The articles in this section all deal with one or more of these important issues.

\section{References}

1. Manchetanz J, Foster J, Bischoff C Meyer BU, Isenberg C, Conrad B. PC based system for an objective quantific tion of manual movement disability fo clinical and scientific purposes. J Biom Eng 1993; 15: 363-70.

2. Aston ML, Wener MH, Thomas RG, Hunder GG, Bloch DA. Applicatiould neural networks to the classificationd giant cell arthritis. Arthritis \& Rheum tism 1994; 37: 760-70.

3. Linnarsson R. Drug interations in pri mary health care. Scand J Prim Healit Care 1993; 11: 181-6.

4. Kahn MG. Clinical databases and criti cal care research. Critical Care Clinic 1994; 10: 37-51.

5. Gorman PN, Ash J, Wykoff L. Can pir mary care physicians' questions be an swered using the medical journal liten ture? Bull Med Libr Assoc 1994; 82 140-6.

6. Klein MS, Ross FV, Adams DL, Gilbet CM. Effect of online literature sarchim on length of stay and patient care cost Acad Med 1994; 69: 489-95.

Address of the author: Hiroshi Takeda, M.D. Department of Medical Information Science Osaka University Medical School,

2-15, Yamada-oka, Suita , Osaka 565, Japan 